\title{
Influence of Polymer Blending on Mechanical and Thermal Properties
}

\author{
Buthaina A. Ibrahim \& Karrer M.Kadum \\ University of Technology, Baghdad, Iraq
}

\begin{abstract}
Polymer blends are capable of providing materials with extended useful properties beyond the range that can be obtained from single polymer equivalents. Blends of polystyrene (PS) and Acrylonitrile-Butadiene-Styrene (ABS) are prepared in different ratios by melt blending technique which was carried out using a single screw extruder. The tensile test and Differential Scanning Calorimetry (DSC) are used to study mechanical and thermal properties. The results from this work show that the mechanical properties for blend system are better than those of pure polymers, also the (DSC) test gives good indications of improving state of miscibility for most blend ratios; there is only one glass transition temperature between the two values of pure polymers.
\end{abstract}

Keywords: Polymer Blend, Mechanical properties, Differential Scanning Calorimetry (DSC)

\section{Introduction}

Polymer blend represents very important field in processing of new materials, which has better properties in comparison with the net polymers. They are significant also from ecological and economical viewpoint. Thus, for example, municipal commingled plastic waste, composed of various immiscible polymers can be recycled by mixing in molten state, and so it can be transformed to the material, which would satisfy the relevant application. High molecular weight polymers show low entropy of mixing (Cimmino, S., Karasz, F. E., Macknight, W. J. 1992). So that, most polymer blends show immiscible systems, whose properties are not only a function of the blend composition but also depend crucially on the degree of dispersion phase particle size, and phase interaction between the components of the blend. Also by controlling the morphology for polymer blend lead to practicability to controlling the polymer blend properties(Datta S., Lohse, D. 1993). Many polymer pairs are not only immiscible but also incompatible. It means that they show high interfacial tension that leads to rough phase structure, poor adhesion at the interface, and therefore poor mechanical properties. Compatibility arises from thermodynamic interaction between the blend constituents, which is a function of their physical and chemical structure (J. W. Barlow and D. R. Paul. 1985). The main reasons lead to creating incompatibles systems that can be summarized as: absence of any specific interaction between their blend constituents, dissimilarity in their structure, and broad differences in their viscosities.

Many articles were devoted to studying the effect of different reasons on the compatibility, miscibility, morphology, and mechanical properties for the polymer blend. Willis and Favis (J. M. Willis and B.D.Favis, 1988) studied the processing and phase morphology relationship of compatibilized polylefin-polyamide blends with the help of an ionomer compatibilizer. They found that there is an abrupt increase in dispersion and interfacial adhesion and a decrease in particle of the dispersed nylon phase. This was reported to be due to specific interaction between nylon and the ionomer at the interface in the blend of polyethylene and nylon similarly. Wycisk R,et.al prepared polymer blend from polyethylene and polystyrene, there are three samples of different molecular weight from polystyrene. They found the morphology of the blends changed where spherical domains of one component, dispersed in continuous matrix of the other, changed to a highly non homogeneous mixture of fibrous or cabbage-like macro-domains of both components. This change in morphology depends on the composition and molecular weight of polystyrene. The aim of the present study is to show the effect of blend ratios on the mechanical properties and study the probability to creating compatible system for the polymer blend. Such system may be satisfied by correct choice of methods of polymer blends preparation, and the best methods for detecting miscibility by Differential Scanning Calorimeter (DSC). If the DSC test detects one glass transition temperature for the polymer blend, then this leads to averment of state of miscibility.

\section{Materials and Method}

PS and ABS are obtained from the Chemical Industries Company, Baghdad. Polystyrene is a thermoplastic substance, which is in solid (glassy) state at room temperature, but flows when heated above its glass transition temperature (for molding or extrusion), and becomes solid again when it cools off. ABS is a heterogeneous thermoplastic, which consists of two phases terpolymer, consisting of dispersed rubbery phases made up of polybutadiene (PB) rubber graft with styrene acrylontrile (SAN) and then dispersed in a continuous plastic of more SAN(M.M.K. Khan, R.F. Liang, R.K. Gupta, S. Agarwal. 2005). 


\section{A) Sample preparations}

Two polymers were mixed and prepared by melt extrusion using single screw extruder in a system of polystyrene / poly(acrylonitrile-butadiene-styrene), (PS /ABS) with different composition ratios as summarized in Table 1.

\section{B) Testing}

a. Tensile test

The tensile test is carried out for both specimens which are prepared by melt extrusion methods, according to ASTM standards using Instron testing machine (model PLTE-329.TSQ) (Figure (3.4)) using cross head speed of $0.02 \mathrm{~mm} / \mathrm{min}$, for specimen prepared by solvent casting, and cross head speed of $0.5 \mathrm{~mm} / \mathrm{min}$ for extrusion specimen. The load applied was $(0-50000) \mathrm{N}$.

\section{b. DSC test}

The measurements of glass transition temperature and melting point were carried out with a Perkin-Elmer differential scanning calorimeter (DSC-6). Throughout the DSC work, samples were encapsulated in aluminum sample pans and placed in the holder. An empty aluminum pan of the same weight as that used with the sample, was used as reference holder. Each holder has its own heater and temperature sensor. With the DSC-6 instrument, nitrogen at flow rate of $20 \mathrm{ml} / \mathrm{min}$. was used as a purge gas.

The glass transition temperatures of the polymer samples were determined with approximately $20 \mathrm{mgm}$ of the $\mathrm{f}$ polymer sample prepared by extrusion methods. Each sample was scanned from initial temperature $\left(30^{\circ} \mathrm{C}\right)$ for the instrument at a rate of $10^{\circ} \mathrm{C} / \mathrm{min}$. This heating rate was chosen to achieve good resolution through DSC test.

\section{Results and Discussion}

A) Tensile test

The results for the pure polymer and the blends of different compositions are summarized in table (2). These results show that:

- The $60 / 40$ blend composition has the highest value of Young's modulus and ultimate strength, while the 70/20 blend composition has the highest value of ultimate strain.

- The 70/30,60/40 and 50/50 blends have better Young's modulus, ultimate strength and ultimate strain than the pure PS or ABS materials.

- The 90/10 and 40/60 blend compositions have very poor mechanical properties, which are worse than those for the pure materials.

- The $80 / 20$ and 20/80 blend compositions show the same mechanical behaviors, i.e. they have Young's modulus and ultimate strain \% higher than those of the pure materials, while the ultimate strengths are the same as that for ABS but slightly less than that for PS.

One of the physical characteristics of immiscible blends is having weaker mechanical properties than pure polymers, although there are some methods that will make immiscible blends stronger and compatible. One of these methods is to process these by mechanical mixing in the melt.

Morton and Hearle(Wei Chen, Gary C. Lickfield and Charles Q. 2004), interpreted the mechanical properties of polymer in terms of molecular motion. They explained the initial modulus in terms of internal stresses. At low applied stress, polymers exhibit high resistance to deformation owing to the secondary bonds between the chains and the geometrical interaction between the molecules chains, which cannot move independently of their neighbors. The small motion of chain segments caused by the applied stress in this range in attributed to the diffusion of short chain segments into the "hole" in the polymer microstructure.

From the results of Young's modulus in table 2 it may be seen that the 90/10 and 40/60 blend compositions incompatibility may be attributed to that during extrusion the solidification of the matrix phase PS before minor phase ABS may result in bad adhesion between two phases. Bad adhesion between two phases results in lower interfacial tension and hence the lower is the internal stress.

The compositions 70/30, 60/40 and 50/50 of PS/ABS blends have mechanical properties that are better than the pure materials, which may be due to their processing under flow in one direction where the minor components form rods instead of spheres as in blending in co-solvents. These rods act like fibers of reinforced composite material, while when the two polymers are roughly in equal amount, they from two co-continuous phases. This means both phases will bear the load if any stress is applied on the material, then it will be stronger. Also it may be that by increasing the amount of ABS, then the styrene proportions within ABS increase. This styrene will be capable of forming chemical bonding with the polystyrene compound during extrusion, which consequently enhances the adhesion between the two phases. This good adhesion of $60 / 40$ blend component shows the highest 
value of strength and less strain \%, which means with better adhesion between the two phases results in greater interfacial tension and hence higher internal stress which will lead to lower extensibility.

\section{B) DSC test results and discussion}

The DSC test in this work was curried out mainly to investigate the effect of PS/ABS blend, extruded with different compositions, on the value of glass transition temperature. The results for pure polymer and blends with different compositions are shown in figure (1, a, b, c, d, e).

Figure (1 a) shows the DSC results for extruded polystyrene (PS), where the glass transition temperature is found to be $119.3^{\circ} \mathrm{C}$. The DSC results for extruded PS/ABS blend samples are shown in figures $1(\mathrm{~b}, \mathrm{c}, \mathrm{d}$ and e); compositions are 80/20,70/30, 60/40 and 50/50 respectively. For these blend compositions, the Tg's are found to be $116.7,115.8,114.8$ and $114.0^{\circ} \mathrm{C}$ respectively. It is observed that the $\mathrm{Tg}$ value decreases with increasing ABS composition. The ABS Tg has been reported elsewhere as $110^{\circ} \mathrm{C}$. The $\mathrm{Tg}$ for the mixtures is calculated by using an approximate relationship between $\mathrm{Tg}$ for mixture and composition as given by the simple (rule of mixture) for binary mixture (Fried J.R. 2005)

$$
\frac{1}{T_{g}}=\frac{W_{1}}{T_{g 1}}+\frac{W_{2}}{T_{g 2}}
$$

Where, $\mathrm{W}_{1}$ and $\mathrm{W}_{2}$ are the weight fraction of two polymers, having $\mathrm{Tg}_{1}$ and $\mathrm{Tg}_{2}$ respectively. From these calculations, the value of $\mathrm{T}_{\mathrm{g}}$ 's for the above compositions are $117.0,116.1,115.2$ and $114.3^{\circ} \mathrm{C}$. From these results, it seems that for all compositions the calculated $\mathrm{T}_{\mathrm{g}}$ 's are slightly higher than those fond by DSC;

$T_{g}($ calc. $)-T_{g}(D S C)=0.3^{\circ} \mathrm{C}$

It is reported that $\mathrm{T}_{\mathrm{g}}$ 's behavior for polymer blends has been used as a measure of miscibility. The appearance of a single $T_{g}$ at a temperature intermediate between the $T_{g}$ 's of pure components is indicative of molecular homogeneity, while the appearance of multiple $\mathrm{T}_{\mathrm{g}}$ 's reflects phase separation of the blend's components (Merfeld,G.D. and Paul D.R. 2000).

\section{Conclusion}

This paper introduces a new study of blends of polystyrene (PS) and Acrylonitrile-Butadiene-Styrene (ABS) that are prepared in different ratios by melt blending technique which was carried out using a single screw extruder. For all these blend ratios (70/30, 60/40 and 50/50) \%

(PS/ABS) the mechanical properties are better than for pure polymer. The (DSC) test averment the state of miscibility by recording one glass transition temperature.

\section{References}

Cimmino, S., Karasz, F. E., Macknight, W. J. (1992). J Polym Sci Polym Phys Ed 1992, 30, 49.

Datta S., Lohse, D. (1993). J. Macromolecules 1993, 26, 2064.

Fried J.R. (2005). "polymer science and technology"2nd edition printice hill,India2005.

http://en.wikipedia.org/wiki/Polystyrene_(disambiguation)

http://pslc.ws/mactest/iblend.htm

http://www.fortus.com/uploadedFiles/North_America/Materials/Files-Materials/Stratasys_ABS.pdf

J. M. Willis and B.D.Favis. (1988). Polym. Eng. Sci., 98(21),1416 (1988).

J. W. Barlow and D. R. Paul. (1985). Polym. Eng. Sci.,21(15), 985 (1981).

M.M.K. Khan, R.F. Liang, R.K. Gupta, S. Agarwal. (2005). Korea-Australia Rheology Journal, Vol. 17, No. 1, March 2005 pp. 1-7.

Merfeld,G.D. and Paul D.R. (2000). "polymer blends :formulation" Vel1,John wileg and sons,Inc,USA,2000.

Wei Chen, Gary C. Lickfield and Charles Q. (2004). Yang polymer 45( 3), 2004, P 1063-1071. 
Table 1. Extrusion procedure of blend preparation

\begin{tabular}{|c|c|c|c|}
\hline $\begin{array}{c}\text { Polymeric } \\
\text { blends percents } \\
\text { for (PS/ABS) }\end{array}$ & \multicolumn{2}{|c|}{ Temperature ${ }^{\mathbf{~} \mathbf{C}}$} & \multirow{2}{*}{$\begin{array}{c}\text { Screw speed } \\
\text { Cycle per min. }\end{array}$} \\
\cline { 2 - 3 }$(100 / 0) \%$ & 200 & 225 & 48 \\
\hline$(90 / 10) \%$ & 190 & 210 & 48 \\
\hline$(80 / 20) \%$ & 190 & 210 & 46 \\
\hline$(70 / 30) \%$ & 180 & 200 & 48 \\
\hline$(60 / 40) \%$ & 170 & 190 & 48 \\
\hline$(50 / 50) \%$ & 170 & 190 & 48 \\
\hline$(40 / 60) \%$ & 140 & 160 & 48 \\
\hline$(20 / 80) \%$ & 130 & 150 & 49 \\
\hline$(0 / 100) \%$ & 130 & 150 & 48 \\
\hline
\end{tabular}

Table 2. Mechanical Properties of PS/ABS Blends

\begin{tabular}{|l|l|l|l|}
\hline $\begin{array}{c}\text { Polymeric } \\
\text { blend ratios } \\
\text { for (PS/ABS) }\end{array}$ & $\begin{array}{c}\text { Young's } \\
\text { modulus } \\
\text { MPa }\end{array}$ & Strain\% & $\begin{array}{c}\text { Tensile } \\
\text { strength } \\
\text { MPa }\end{array}$ \\
\hline$(100 / 0)$ & 12.69 & 3.25 & 39.5 \\
\hline$(90 / 10)$ & 10.6 & 3.4 & 19.6 \\
\hline$(80 / 20)$ & 17.63 & 11.25 & 35.3 \\
\hline$(70 / 30)$ & 17.51 & 20 & 43.98 \\
\hline$(60 / 40)$ & 21.1 & 10.1 & 50.8 \\
\hline$(50 / 50)$ & 17.9 & 13.2 & 45.68 \\
\hline$(40 / 60)$ & 10.1 & 8.5 & 18.9 \\
\hline$(20 / 80)$ & 16.7 & 17 & 34.68 \\
\hline$(0 / 100)$ & 14.42 & 13 & 35.8 \\
\hline
\end{tabular}

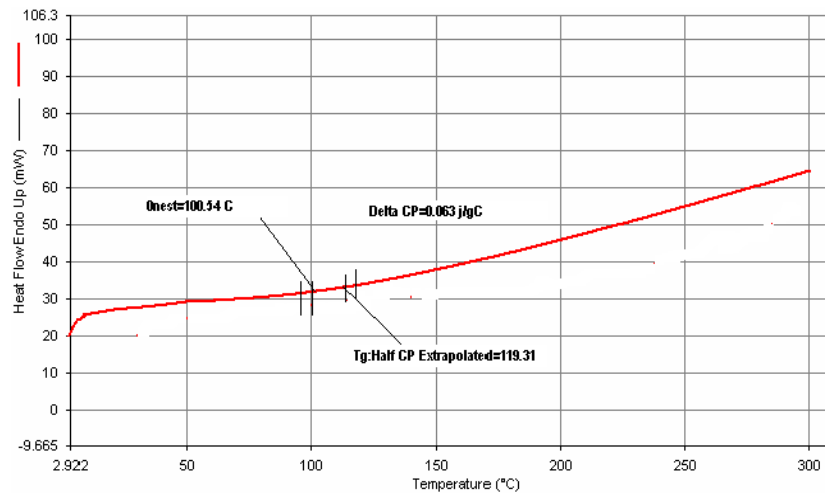

Figure $(1, a)$. glass transition temperature for pure polystyrene. 


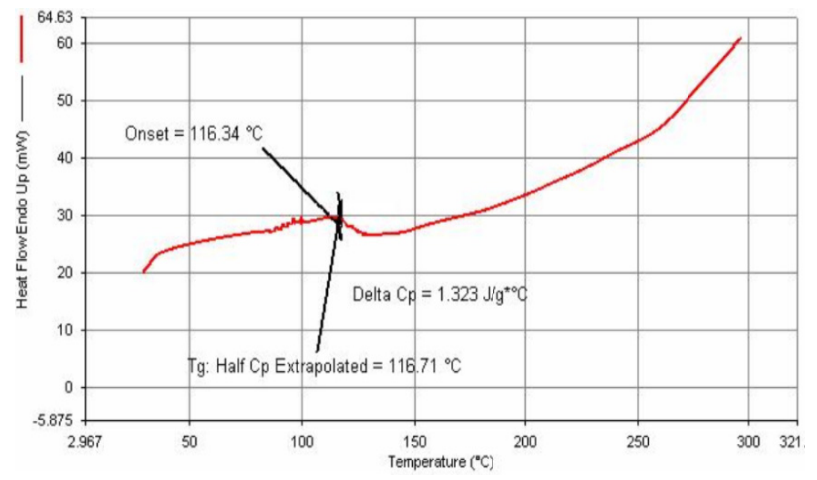

Figure $(1, b)$. glass transition temperature for sample of $(80 / 20)$ PS/ABS blends.

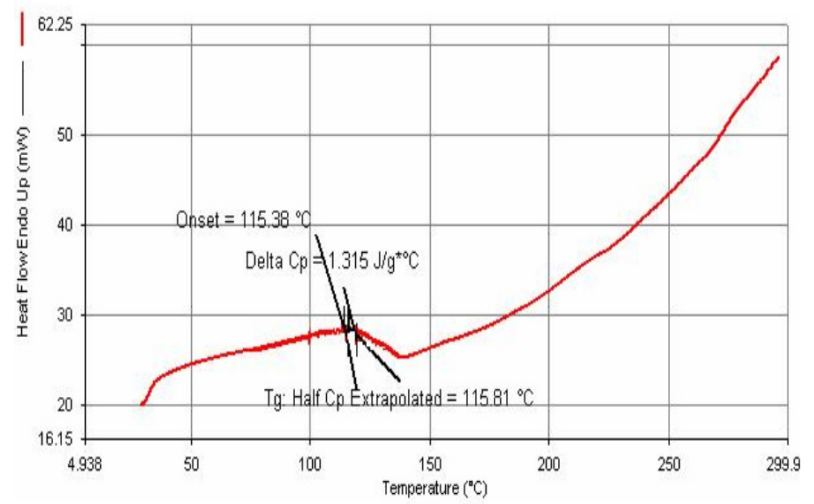

Figure $(1, \mathrm{c})$. glass transition temperature for sample of (70/30) PS/ABS blends.

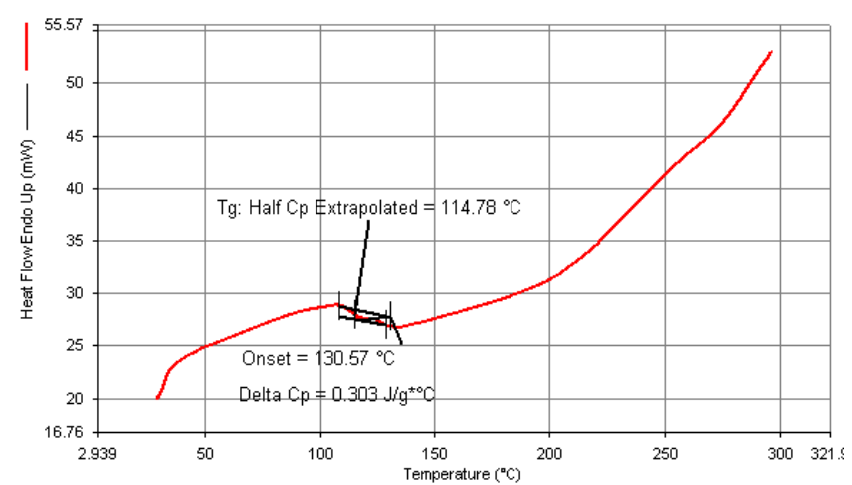

Figure $(1, d)$. glass transition temperature for sample of (60/40) PS/ABS blends.

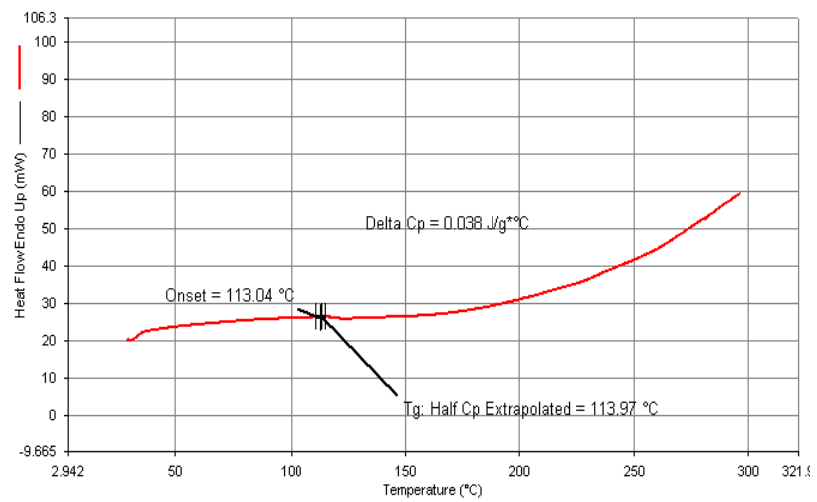

Figure $(1, \mathrm{e})$. glass transition temperature for sample of(50/50) PS/ABS blends. 